Теорія Ймовір. та Матем. Статист. Вип. 78, 2008
Theor. Probability and Math. Statist.

No. 78, 2009, Pages 37-47 S 0094-9000(09)00760-1

Article electronically published on August 4, 2009

\title{
ESTIMATION FOR THE DISCRETELY OBSERVED TELEGRAPH PROCESS
}

UDC 519.21

\author{
S. M. IACUS AND N. YOSHIDA
}

\begin{abstract}
The telegraph process $\{X(t), t>0\}$ is supposed to be observed at $n+1$ equidistant time points $t_{i}=i \Delta_{n}, i=0,1, \ldots, n$. The unknown value of $\lambda$, the underlying rate of the Poisson process, is a parameter to be estimated. The asymptotic framework considered is the following: $\Delta_{n} \rightarrow 0, n \Delta_{n}=T \rightarrow \infty$ as $n \rightarrow \infty$. We show that previously proposed moment type estimators are consistent and asymptotically normal but not efficient. We study further an approximated moment type estimator which is still not efficient but comes in explicit form. For this estimator the additional assumption $n \Delta_{n}^{3} \rightarrow 0$ is required in order to obtain asymptotic normality. Finally, we propose a new estimator which is consistent, asymptotically normal and asymptotically efficient under no additional hypotheses.
\end{abstract}

\section{INTRODUCTION}

The telegraph process (see Goldstein [8] and Kac [12]) models a random motion with finite velocity, and it is usually proposed as an alternative to diffusion models. The process describes the position of a particle moving on the real line, alternatively with constant velocity $+v$ or $-v$. The changes of direction are governed by a homogeneous Poisson process with rate $\lambda>0$. The telegraph process or telegrapher's process is defined as

$$
X(t)=x_{0}+V(0) \int_{0}^{t}(-1)^{N(s)} d s, \quad t>0
$$

where $V(0)$ is the initial velocity taking values $\pm v$ with equal probability and independently of the Poisson process $\{N(t), t>0\}$. Many authors analyzed probabilistic properties of the process over the years (see e.g. Orsingher [16] and [17; Pinsky [18; Fong and Kanno [7; Stadje and Zacks [21]). Di Crescenzo and Pellerey [4] proposed the geometric telegraph process as a model to describe the dynamics of the price of risky assets, i.e. $S(t)=s_{0} \exp \{\alpha t+\sigma X(t)\}, t>0$, where $X(t)$ replaces the standard Brownian motion of the original Black-Scholes [1] and Merton [15] model. Conversely to the geometric Brownian motion, given that $X(t)$ is of bounded variation, so is the geometric telegraph process. This seems a realistic way to model paths of assets in the financial markets. Mazza and Rullière 14 linked the process (11) and the ruin processes in the context of risk theory. Di Masi, Y. Kabanov, and W. Runggaldier [5] proposed to model the volatility of financial markets in terms of the telegraph process. Ratanov [19, 20]

2000 Mathematics Subject Classification. Primary 60K99; Secondary 62M99.

Key words and phrases. Telegraph process, discretely observed process, inference for stochastic processes.

The work of the first author was supported by JSPS (Japan Society for the Promotion of Science) Program FY2006, grant ID No. S06174. He is also thankful to the Graduate School of Mathematical Sciences, University of Tokyo as host research institute for the JSPS Program.

(C)2009 American Mathematical Society 
proposed to model financial markets using a telegraph process with two intensities $\lambda_{ \pm}$ and two velocities $v_{ \pm}$. The telegraph process has also been used in ecology to model population dynamics (see Holmes et al. [10]) and the displacement of wild animals on the soil. In particular, this model is chosen since it preserves the property of animals to move at finite velocity and for a certain period along one direction (see e.g. Holmes [9], for an account).

It is worth mentioning that, up to now, only a few references about estimation problems for the telegrapher's process are known. Yao 22 considers the problem of state estimation of the telegrapher's process under white noise perturbation and studies performance of nonlinear filters. Iacus [11] considers the estimation of the parameter $\theta$ of the non-constant rate $\lambda_{\theta}(t)$ from continuous observations of the process. More recently, De Gregorio and Iacus 2 proposed pseudo-maximum likelihood and moment-based estimators for the telegraph process under discrete observations on a fixed time interval $[0, T]$ when the process is observed with a mesh decreasing to zero.

The aim of this paper is the estimation of the parameter $\lambda$ when $\{X(t), 0 \leq t \leq T\}$ is observed at equidistant times $0=t_{0}<\cdots<t_{n}$. We assume that $t_{i}=i \Delta_{n}, i=0, \ldots, n$; hence $n \Delta_{n}=T$. The asymptotic framework is the following: $\Delta_{n} \rightarrow 0$ and $n \Delta_{n}=T \rightarrow \infty$ as $n \rightarrow \infty$.

If the telegraph process $X(t)$ is observed continuously, then $N(T) / T$ is the optimal estimator of the parameter $\lambda$ and the statistical experiment is equivalent to the one of the observation of the whole Poisson process on $[0, T]$ (see e.g. Kutoyants [13). This situation also corresponds to the limiting experiment in our asymptotic framework.

The paper is organized as follows. Section 2 reviews some results on the telegraph process and presents an explicit formula of the moments of the process. This result is interesting in itself because it gives new information about this model. Section 3 presents estimators previously introduced in the literature such as pseudo-maximum likelihood estimators and moment type estimators. In particular, for the moment type estimator it is shown that it is consistent and asymptotically normal but not efficient. Another approximated moment type estimator is given in explicit form, and it is shown that the estimator is consistent and asymptotically Gaussian but still not efficient. Finally, Section 4 presents a new estimator which is consistent, asymptotically Gaussian and asymptotically efficient.

\section{Moments of the telegraph PRocess}

The process $X(t)$ is not Markovian. Conversely, the two-dimensional process

$$
(X(t), V(t)), \quad V(t)=V(0)(-1)^{N(t)}
$$

has the Markov property but a scheme of observation in which one is able to observe both the position and the velocity of the process at discrete time instants is not admissible, so statistical procedures should rely only on the observation of the $X(t)$ component. The distribution of the position of the particle at time $t$, i.e. the distribution of $X(t)$, is a mixture of continuous and discrete components. This distribution has been obtained by Goldstein [8], Orsingher [17, and Pinsky [18, with different techniques. The transition density is given by

$$
\begin{aligned}
p\left(x, t ; x_{0}, 0\right)= & \frac{e^{-\lambda t}}{2 v}\left\{\lambda I_{0}\left(\frac{\lambda}{v} \sqrt{v^{2} t^{2}-\left(x-x_{0}\right)^{2}}\right)\right. \\
& \left.+\frac{\partial}{\partial t} I_{0}\left(\frac{\lambda}{v} \sqrt{v^{2} t^{2}-\left(x-x_{0}\right)^{2}}\right)\right\} \mathbf{1}_{\left\{\left|x-x_{0}\right|<v t\right\}} \\
+ & \frac{e^{-\lambda t}}{2}\left\{\delta\left(x-x_{0}-v t\right)+\delta\left(x-x_{0}+v t\right)\right\}
\end{aligned}
$$


for any $\left|x-x_{0}\right| \leq v t$, where $I_{\nu}(x)$ is the modified Bessel function of order $\nu, \mathbf{1}_{A}$ is the indicator function of a set $A$ and $\delta$ is the Dirac delta function. Note that the last term in equation (2) represents the singular component of the distribution of (11). Indeed, if no Poisson event occurs in the interval $[0, t]$, we have that

$$
\mathrm{P}\{X(t)=+v t\}=\mathrm{P}\{X(t)=-v t\}=\frac{1}{2} e^{-\lambda t}
$$

In the sequel, we consider the telegraph process starting from the origin at $t=0$, i.e., $X(0)=x_{0}=0$, unless otherwise mentioned. The first two moments of the process are well known:

$$
\mathrm{E} X(t)=0
$$

and

$$
\mathrm{E}\{X(t)\}^{2}=\frac{v^{2}}{\lambda}\left(t-\frac{1-e^{-2 \lambda t}}{2 \lambda}\right)
$$

(see Orsingher [17]). Di Crescenzo and Martinucci [3] derived the moment generating function for the telegraph process; i.e., for all $s \in \mathbb{R}$ and $t \geq 0$,

$$
\mathrm{E}\left\{\mathrm{e}^{s X(t)}\right\}=e^{-\lambda t}\left\{\cosh \left(t \sqrt{\lambda^{2}+s^{2} v^{2}}\right)+\frac{\lambda}{\sqrt{\lambda^{2}+s^{2} v^{2}}} \sinh \left(t \sqrt{\lambda^{2}+s^{2} v^{2}}\right)\right\} .
$$

Theorem 2.1 below gives the general explicit derivation of the moments of the telegraph process. In some sense, the above and the following result integrate Section 3 of Orsingher [17. It is trivially true that the odd moments of the telegraph process are all zero, hence we present the formula for the moments of even order.

Theorem 2.1. For every positive integer $q$,

$$
\mathrm{E}\{X(t)\}^{2 q}=(v t)^{2 q}\left(\frac{2}{\lambda t}\right)^{q-\frac{1}{2}} \Gamma\left(q+\frac{1}{2}\right)\left\{I_{q+\frac{1}{2}}(\lambda t)+I_{q-\frac{1}{2}}(\lambda t)\right\} e^{-\lambda t} .
$$

Proof. We start by rewriting the $2 q$-th moment of $X(t)$ as the sum of the two terms emerging from the discrete and the absolutely continuous part of its density (2). Therefore we have the following representation:

$$
\mathrm{E}\{X(t)\}^{2 q}=\int_{-v t}^{+v t} x^{2 q} p(x, t ; 0,0) d x+(v t)^{2 q} e^{-\lambda t} .
$$

The term $e^{-\lambda t}$ will also appear in the above integral, so we consider separately the two identities

$$
\frac{1}{2} \frac{\lambda}{v} \int_{-v t}^{+v t} x^{2 q} I_{0}\left(\frac{\lambda}{v} \sqrt{v^{2} t^{2}-x^{2}}\right) d x=\left(\frac{2}{\lambda t}\right)^{q-\frac{1}{2}}(v t)^{2 q} \Gamma\left(q+\frac{1}{2}\right) I_{q+\frac{1}{2}}(\lambda t)
$$

and

(9) $\frac{1}{2} \lambda t \int_{-v t}^{+v t} x^{2 q} \frac{I_{1}\left(\frac{\lambda}{v} \sqrt{v^{2} t^{2}-x^{2}}\right)}{\sqrt{v^{2} t^{2}-x^{2}}} d x=(v t)^{2 q}\left\{\Gamma\left(q+\frac{1}{2}\right)\left(\frac{2}{\lambda t}\right)^{q-\frac{1}{2}} I_{q-\frac{1}{2}}(\lambda t)-1\right\}$.

After multiplication of both (8) and (9) by the factor $e^{-\lambda t}$, direct substitution in (7) gives the result of the theorem. So we need to prove the above identities and we start 
with formula (8):

$$
\begin{aligned}
\frac{1}{2} \frac{\lambda}{v} \int_{-v t}^{+v t} x^{2 q} & I_{0}\left(\frac{\lambda}{v} \sqrt{v^{2} t^{2}-x^{2}}\right) d x=\frac{1}{2} \frac{\lambda}{v} \int_{-v t}^{+v t} x^{2 q} \sum_{k=0}^{\infty} \frac{1}{(k !)^{2}}\left(\frac{\lambda}{2 v} \sqrt{v^{2} t^{2}-x^{2}}\right)^{2 k} d x \\
& =\frac{1}{2} \frac{\lambda}{v} \sum_{k=0}^{\infty} \frac{1}{(k !)^{2}}\left(\frac{\lambda}{2 v}\right)^{2 k} \int_{-v t}^{+v t} x^{2 q}\left(v^{2} t^{2}-x^{2}\right)^{k} d x \\
& =\frac{1}{2} \frac{\lambda}{v} \sum_{k=0}^{\infty} \frac{1}{(k !)^{2}}\left(\frac{\lambda}{2 v}\right)^{2 k}(v t)^{2(q+k)+1} \mathrm{~B}\left(k+1, q+\frac{1}{2}\right) \\
& =\left(\frac{2}{\lambda t}\right)^{q-\frac{1}{2}}(v t)^{2 q} \Gamma\left(q+\frac{1}{2}\right) I_{q+\frac{1}{2}}(\lambda t),
\end{aligned}
$$

where we used the relations $\mathrm{B}(a, b)=\Gamma(a) \Gamma(b) / \Gamma(a+b), \Gamma(x)=\int_{0}^{\infty} e^{-t} t^{x-1} d t, n !=$ $\Gamma(n+1)$,

$$
I_{\nu}(x)=\sum_{k=0}^{\infty} \frac{1}{\Gamma(k+1+\nu) k !}\left(\frac{x}{2}\right)^{2 k+\nu}
$$

and

$$
\int_{-v t}^{+v t} x^{2 q}\left(v^{2} t^{2}-x^{2}\right)^{k} d x=(v t)^{2(k+q)+1} \mathrm{~B}\left(k+1, q+\frac{1}{2}\right) .
$$

We now calculate (9):

$$
\begin{aligned}
\frac{1}{2} \lambda t & \int_{-v t}^{+v t} x^{2 q} \frac{I_{1}\left(\frac{\lambda}{v} \sqrt{v^{2} t^{2}-x^{2}}\right)}{\sqrt{v^{2} t^{2}-x^{2}}} d x \\
& =\frac{1}{2} \lambda t \sum_{k=0}^{\infty} \frac{1}{\Gamma(k+2) k !}\left(\frac{\lambda}{2 v}\right)^{2 k+1} \int_{-v t}^{+v t} x^{2 q}\left(v^{2} t^{2}-x^{2}\right)^{k} d x \\
& =\frac{1}{2} \lambda t \sum_{k=0}^{\infty} \frac{1}{\Gamma(k+2) k !}\left(\frac{\lambda}{2 v}\right)^{2 k+1}(v t)^{2(k+q)+1} \mathrm{~B}\left(k+1, q+\frac{1}{2}\right) \\
& =\frac{1}{2}(v t)^{2 q} \Gamma\left(q+\frac{1}{2}\right)\left\{2^{q+\frac{1}{2}}(\lambda t)^{\frac{1}{2}-q} I_{q-\frac{1}{2}}(\lambda t)-\frac{(1+2 q)}{\Gamma\left(\frac{3}{2}+q\right)}\right\} \\
& =(v t)^{2 q}\left\{\Gamma\left(q+\frac{1}{2}\right)\left(\frac{2}{\lambda t}\right)^{q-\frac{1}{2}} I_{q-\frac{1}{2}}(\lambda t)-1\right\}
\end{aligned}
$$

because $\Gamma(q+1 / 2) / \Gamma(q+3 / 2)=2 /(1+2 q)$.

Remark 2.1. The modified Bessel functions admit the following expansion:

$$
I_{\nu}(x)=\frac{1}{\Gamma(\nu+1)}\left(\frac{x}{2}\right)^{\nu}\left(1+\frac{x^{2}}{4(\nu+1)}+\frac{x^{4}}{32(\nu+1)(\nu+2)}+\cdots\right),
$$

from which we obtain that $\mathrm{E}\{X(t)\}^{2 q}$ is of order $t^{2 q}$ as $t \rightarrow 0$. The following expansion, for $t \rightarrow 0$, will be useful in the following:

$$
\begin{aligned}
& \mathrm{E}\{X(t)\}^{2}=v^{2} t^{2}-\frac{2}{3} v^{2} \lambda t^{3}+\frac{1}{3} v^{2} \lambda^{2} t^{4}+o\left(t^{4}\right), \\
& \mathrm{E}\{X(t)\}^{4}=v^{4} t^{4}-\frac{4}{5} v^{4} \lambda t^{5}+\frac{2}{5} v^{4} \lambda^{2} t^{6}+o\left(t^{6}\right), \\
& \mathrm{E}\{X(t)\}^{6}=v^{6} t^{6}-\frac{6}{7} v^{6} \lambda t^{7}+\frac{3}{7} v^{6} \lambda^{2} t^{8}+o\left(t^{8}\right) .
\end{aligned}
$$


We now check that for $q=1$ we recover formula (4), which has been derived in two different ways in Orsingher [17]. Indeed, for $q=1$ we have

$$
\mathrm{E}\{X(t)\}^{2}=(v t)^{2}\left(\frac{2}{\lambda t}\right)^{\frac{1}{2}} \frac{\sqrt{\pi}}{2} e^{-\lambda t}\left\{I_{\frac{3}{2}}(\lambda t)+I_{\frac{1}{2}}(\lambda t)\right\}
$$

and noticing that

$$
I_{\frac{1}{2}}(x)=\sqrt{\frac{2}{\pi}} \frac{\sinh (x)}{\sqrt{x}}, \quad I_{\frac{3}{2}}(x)=\sqrt{\frac{2}{\pi}} \frac{x \cosh (x)-\sinh (x)}{\sqrt{x^{3}}},
$$

direct substitution gives (41).

Remark 2.2. The formula of the fourth moment also has a relatively simple expression, so we present it here. To derive the result, it is useful to know that

$$
I_{\frac{5}{2}}(x)=\sqrt{\frac{2}{\pi}} \frac{\left(x^{2}+3\right) \sinh (x)-3 x \cosh (x)}{\sqrt{x^{5}}} .
$$

Thus,

$$
\begin{aligned}
\mathrm{E}\{X(t)\}^{4} & =(v t)^{4}\left(\frac{2}{\lambda t}\right)^{\frac{3}{2}} \frac{3}{4} \sqrt{\pi}\left\{I_{\frac{5}{2}}(\lambda t)+I_{\frac{3}{2}}(\lambda t)\right\} e^{-\lambda t} \\
& =3\left(\frac{v}{\lambda}\right)^{4} e^{-\lambda t}\{\lambda t(\lambda t-3) \cosh (\lambda t)+(3+\lambda t(\lambda t-1)) \sinh (\lambda t)\} .
\end{aligned}
$$

One can easily calculate the same moments starting from the moment generating function in (5) in the usual way, i.e.

$$
\mathrm{E}\{X(t)\}^{k}=\left.\frac{\partial^{k}}{\partial s^{k}} \mathrm{E}\left\{e^{s X(t)}\right\}\right|_{s=0}
$$

but formula (6) seems a little easier to use.

\section{Previous Results on the estimation of $\lambda$ And ASymptotic properties}

As mentioned in the Introduction, we assume that the telegraph process

$$
\{X(t), 0 \leq t \leq T\},
$$

with $X(0)=x_{0}=0$, is observed only at discrete times $0<t_{1}<\cdots<t_{n}=T$, with $t_{i}=i \Delta_{n}, i=0, \ldots, n$; hence $n \Delta_{n}=T$. We use the following notation to simplify the formulas: $X\left(t_{i}\right)=X\left(i \Delta_{n}\right)=X_{i}$. The interest is in the estimation of the parameter $\lambda$ whilst $v$ is assumed to be known. If the whole trajectory can be observed, $\lambda$ can be estimated by $N(T) / T$, where $N(T)$ is the number of Poisson events counted in $[0, T]$ or the number of times the process switches its velocity in $[0, T]$. The estimation of $v$ is always an uninteresting problem as, if there are no switchings in $\left((i-1) \Delta_{n}, i \Delta_{n}\right]$, then $X_{i}-X_{i-1}=v \Delta_{n}$; hence if $\Delta_{n}$ is sufficiently small, there is a high probability to observe $N\left(t_{i+1}\right)-N\left(t_{i}\right)=0$ and then $v$ can be estimated (actually calculated) without error.

We now review some estimators for this process already available in the literature and study some new properties of one of them. De Gregorio and Iacus 2] considered the 
following approximated likelihood:

$$
\begin{aligned}
L_{n}(\lambda) & =L_{n}\left(\lambda \mid X_{0}, X_{1}, \ldots, X_{n}\right)=\prod_{i=1}^{n} p\left(X_{i}, \Delta_{n} ; X_{i-1}, t_{i-1}\right) \\
= & \prod_{i=1}^{n}\left\{\frac{e^{-\lambda \Delta_{n}}}{2 v}\left\{\lambda I_{0}\left(\frac{\lambda}{v} \sqrt{u_{n, i}}\right)+\frac{v \lambda \Delta_{n} I_{1}\left(\frac{\lambda}{v} \sqrt{u_{n, i}}\right)}{\sqrt{u_{n, i}}}\right\} \mathbf{1}_{\left\{u_{n, i}>0\right\}}\right. \\
& \left.+\frac{e^{-\lambda \Delta_{n}}}{2} \delta\left(u_{n, i}\right)\right\},
\end{aligned}
$$

where $u_{n, i}=u_{n}\left(X_{i}, X_{i-1}\right)=v^{2} \Delta_{n}^{2}-\left(X_{i}-X_{i-1}\right)^{2}$. The density $p\left(X_{i}, \Delta_{n} ; X_{i-1}, t_{i-1}\right)$ appearing in (13) is a version of formula (2), and it is the probability law of a telegraph process initially located in $X_{i-1}$ that reaches the position $X_{i}$ at time $t_{i}$. The above approximate likelihood is just the product of the laws of the increments $X_{i}-X_{i-1}$ which are considered as if they were $n$ independent copies of the process $X\left(\Delta_{n}\right)$. In this sense (13) is only an approximate likelihood function. The increments $\eta_{i}$ can be expressed as follows:

$$
\eta_{i}=X_{i}-X_{i-1}=V(0) \int_{t_{i-1}}^{t_{i}}(-1)^{N(s)} d s=V(0)(-1)^{N\left(t_{i-1}\right)} \int_{t_{i-1}}^{t_{i}}(-1)^{N(s)-N\left(t_{i-1}\right)} d s,
$$

and they are stationary but not independent. Conversely, the squared increments

$$
\eta_{i}^{2}=v^{2}\left(\int_{t_{i-1}}^{t_{i}}(-1)^{N(s)-N\left(t_{i-1}\right)} d s\right)^{2}
$$

(or the absolute increments $\left|\eta_{i}\right|$ ) are independent. In their paper, the authors proposed the following estimator:

$$
\hat{\lambda}_{n}=\arg \max _{\lambda>0} L_{n}(\lambda)
$$

The estimator is proved to exist uniquely (not so evident given the uncommon form of $L_{n}$ ) and such that $\hat{\lambda}_{n} \rightarrow N(T) / T$ under the condition $n \Delta_{n}=T, \Delta_{n} \rightarrow 0$ as $n \rightarrow \infty$ but $T$ fixed. The limiting estimator $N(T) / T$ is the natural estimator, but $\hat{\lambda}_{n}$ is not consistent for all values of $\lambda$ because time $T$ is fixed. In the same paper, the authors present numerical results about a least squares estimator of the following form:

$$
\check{\lambda}_{n}=\arg \min _{\lambda>0}\left\{\frac{1}{n} \sum_{i=1}^{n} \eta_{i}^{2}-\frac{v^{2}}{\lambda}\left(\Delta_{n}-\frac{1-e^{-2 \lambda \Delta_{n}}}{2 \lambda}\right)\right\}^{2} .
$$

In order to have consistency and asymptotic normality of estimators (14) and (15), it is necessary to consider the asymptotics as $n \Delta_{n}=T \rightarrow \infty$. The asymptotic minimal variance of all the estimators for the continuous time experiment is the value of $\lambda$ itself because, as said, it is just the problem of estimating the intensity of a homogeneous Poisson process. We will prove that the estimator $\check{\lambda}_{n}$ is a true moment type estimator which is consistent and asymptotically Gaussian but not efficient because its asymptotic variance is $\frac{6}{5} \lambda>\lambda$. The estimator $\check{\lambda}_{n}$ is given in implicit form. Therefore we also study an approximate moment type estimator which is given in explicit form and prove that it is consistent and asymptotically normal (under the additional condition $n \Delta_{n}^{3} \rightarrow 0$ ) but still not efficient. A new asymptotically efficient estimator will be presented in Section 4 . 
3.1. The moment type estimator. Consider the original estimator in (15). The statistic

$$
V_{n}=\frac{1}{n} \sum_{i=1}^{n} \frac{\eta_{i}^{2}}{\Delta_{n}^{2}}
$$

is an unbiased estimator of

$$
g_{n}(\lambda)=\frac{v^{2}}{\lambda \Delta_{n}^{2}}\left(\Delta_{n}-\frac{1-e^{-2 \lambda \Delta_{n}}}{2 \lambda}\right) .
$$

Observe now that $g_{n}(\lambda)$ is a monotone decreasing function of $\lambda$ such that

$$
\lim _{\lambda \rightarrow 0} g_{n}(\lambda)=v^{2}, \quad \lim _{\lambda \rightarrow \infty} g_{n}(\lambda)=0 .
$$

On the other hand, $V_{n}$ is in $\left[0, v^{2}\right]$ a.s.; hence the minimal value of (15) $\check{\lambda}_{n}$ is also a unique solution to the equation

$$
\frac{1}{n} \sum_{i=1}^{n} \eta_{i}^{2}-\frac{v^{2}}{\lambda}\left(\Delta_{n}-\frac{1-e^{-2 \lambda \Delta_{n}}}{2 \lambda}\right)=0
$$

which means that $\check{\lambda}_{n}$ is a true moment type estimator. Let $\lambda_{0}$ denote the true value of the parameter $\lambda$, and let $\mathrm{E}_{0}$ and $\mathbf{V}_{0}$ indicate respectively the expected value and the variance operator under $\mathrm{P}_{0}$, the law corresponding to the true value of the parameter $\lambda=\lambda_{0}$.

Theorem 3.1. Let $\check{\lambda}_{n}$ be the moment type estimator satisfying (16) and suppose that $n \Delta_{n} \rightarrow \infty, \Delta_{n} \rightarrow 0$ as $n \rightarrow \infty$. Then, $\check{\lambda}_{n}$ is a consistent estimator of $\lambda_{0}$ and

$$
\sqrt{n \Delta_{n}}\left(\check{\lambda}_{n}-\lambda_{0}\right) \stackrel{d}{\rightarrow} N\left(0, \frac{6}{5} \lambda_{0}\right)
$$

as $n \rightarrow \infty$, where $\stackrel{d}{\rightarrow}$ denotes the convergence in distribution.

Proof. From (10), (11), and (12), we have

$$
\begin{gathered}
\mathrm{E}\left\{\eta_{i}^{2}\right\}=\mathrm{E}\left(X_{i}-X_{i-1}\right)^{2}=v^{2} \Delta_{n}^{2}-\frac{2}{3} v^{2} \lambda \Delta_{n}^{3}+O\left(\Delta_{n}^{4}\right) \\
\mathrm{E}\left\{\eta_{i}^{4}\right\}=v^{4} \Delta_{n}^{4}-\frac{4}{5} v^{4} \lambda \Delta_{n}^{5}+O\left(\Delta_{n}^{6}\right) \\
\mathrm{E}\left\{\eta_{i}^{6}\right\}=O\left(\Delta_{n}^{6}\right)
\end{gathered}
$$

so that

$$
\mathbf{V}_{0}\left\{\eta_{i}^{2}\right\}=\mathrm{E}_{0} \eta_{i}^{4}-\left(\mathrm{E}_{0}\left\{\eta_{i}^{2}\right\}\right)^{2}=\frac{8}{15} v^{4} \lambda_{0} \Delta_{n}^{5}+O\left(\Delta_{n}^{6}\right) .
$$

Therefore, by the central limit theorem,

$$
\sqrt{\frac{n}{\Delta_{n}}}\left(V_{n}-g_{n}\left(\lambda_{0}\right)\right) \stackrel{d}{\rightarrow} N\left(0, \frac{8}{15} v^{4} \lambda_{0}\right)
$$

as $n \rightarrow \infty$; the Lindeberg condition is obviously satisfied, since $\left|\eta_{i}^{2} / \Delta_{n}^{2}\right|, g_{n}(\lambda) \leq v^{2}$, which follows from the representation

$$
g_{n}(\lambda)=2 v^{2} \int_{0}^{1}(1-s) e^{-2 \lambda \Delta_{n} s} d s .
$$

For $\varepsilon>0$, let

$$
\gamma(\varepsilon)=2 v^{2} \int_{0}^{1}(1-s) e^{-2 \varepsilon s} d s<v^{2} .
$$


Then

$$
\begin{aligned}
\mathrm{P}_{0}\left\{\check{\lambda}_{n}>\varepsilon \Delta_{n}^{-1}\right\} & \leq \mathrm{P}_{0}\left\{g_{n}\left(\check{\lambda}_{n}\right)<\gamma(\varepsilon)\right\}=\mathrm{P}_{0}\left\{V_{n}<\gamma(\varepsilon)\right\} \\
& \leq \mathrm{P}_{0}\left\{\left|V_{n}-g_{n}\left(\lambda_{0}\right)\right|>\frac{v^{2}-\gamma(\varepsilon)}{2}\right\} \quad \text { (for large } n \text { ) } \\
& \rightarrow 0
\end{aligned}
$$

as $n \rightarrow \infty$, since $\left|V_{n}-g_{n}\left(\lambda_{0}\right)\right| \stackrel{p}{\rightarrow} 0$ due to (17), so that

$$
\check{\lambda}_{n} \Delta_{n} \stackrel{p}{\rightarrow} 0 \text {. }
$$

Let $h_{n}(\lambda)=-\Delta_{n}^{-1} g_{n}^{\prime}(\lambda)$. Then $h_{n}(\lambda)=\delta\left(\lambda \Delta_{n}\right)$ with

$$
\delta(t)=4 v^{2} \int_{0}^{1} s(1-s) e^{-2 t s} d s .
$$

Set

$$
\tilde{V}_{n}(s)=g_{n}\left(\lambda_{0}\right)+s\left(V_{n}-g_{n}\left(\lambda_{0}\right)\right) .
$$

Since $\tilde{V}_{n}(s)$ is between $g_{n}\left(\lambda_{0}\right)$ and $V_{n}, g_{n}^{-1}\left(\tilde{V}_{n}(s)\right)$ is between $\lambda_{0}$ and $\check{\lambda}_{n}$. By (18),

$$
\Gamma_{n}:=\int_{0}^{1}\left[h_{n}\left(g_{n}^{-1}\left(\tilde{V}_{n}(s)\right)\right)\right]^{-1} d s \stackrel{p}{\rightarrow}\left[4 v^{2} \int_{0}^{1} s(1-s) d s\right]^{-1}=\frac{3}{2 v^{2}} .
$$

Finally,

$$
\begin{aligned}
\sqrt{n \Delta_{n}}\left(\check{\lambda}_{n}-\lambda_{0}\right) & =\sqrt{n \Delta_{n}}\left(g_{n}^{-1}\left(V_{n}\right)-g_{n}^{-1}\left(g_{n}\left(\lambda_{0}\right)\right)\right)=-\sqrt{\frac{n}{\Delta_{n}}}\left(V_{n}-g_{n}\left(\lambda_{0}\right)\right) \Gamma_{n} \\
& \stackrel{d}{\rightarrow} N\left(0, \frac{6}{5} \lambda_{0}\right)
\end{aligned}
$$

as $n \rightarrow \infty$.

3.2. An approximate (but explicit) moment type estimator. Consider again (4). Some algebra, or Remark 2.1, gives the following expansion:

$$
\begin{aligned}
\mathrm{E}\left\{X_{i}-X_{i-1}\right\}^{2} & =\frac{v^{2}}{\lambda}\left(\Delta_{n}-\frac{1-e^{-2 \lambda \Delta_{n}}}{2 \lambda}\right) \\
& =\frac{v^{2}}{\lambda}\left(\Delta_{n}-\frac{2 \lambda \Delta_{n}-\frac{1}{2}\left(-2 \lambda \Delta_{n}\right)^{2}-\frac{1}{6}\left(-2 \lambda \Delta_{n}\right)^{3}+o\left(\Delta_{n}^{3}\right)}{2 \lambda}\right) \\
& =v^{2} \Delta_{n}^{2}-\frac{2}{3} v^{2} \lambda \Delta_{n}^{3}+o\left(\Delta_{n}^{3}\right) .
\end{aligned}
$$

Therefore, an approximate moment type estimator is the following:

$$
\lambda_{n}^{*}=\frac{3}{2} \frac{1}{n v^{2} \Delta_{n}^{3}} \sum_{i=1}^{n}\left\{v^{2} \Delta_{n}^{2}-\left(X_{i}-X_{i-1}\right)^{2}\right\}=\frac{3}{2} \frac{1}{n \Delta_{n}} \sum_{i=1}^{n}\left\{1-\frac{\eta_{i}^{2}}{v^{2} \Delta_{n}^{2}}\right\}
$$

and $\lambda_{n}^{*}$ is a weighted sum of the independent random variables $\eta_{i}^{2}$.

Note that $v^{2} \Delta_{n}^{2}-\left(X_{i}-X_{i-1}\right)^{2}$ is exactly zero if no Poisson event occurs in the time interval $\left(t_{i}, t_{i+1}\right]$. This fact will be used to evaluate expected values of related quantities in the following. This estimator is qualitatively not different from the estimator $\check{\lambda}_{n}$ in equation (15); we assume the additional condition $n \Delta_{n}^{3} \rightarrow 0$ in order to obtain asymptotic normality. This hypothesis has also been used in the "high frequency" sampling for discretely observed diffusion processes (see e.g. Florens-Zmirou [6]; Yoshida [23]). 
Theorem 3.2. Under the condition $\Delta_{n} \rightarrow 0, n \Delta_{n} \rightarrow \infty$ as $n \rightarrow \infty$ the statistic $\lambda_{n}^{*}$ in (19) is a consistent estimator of $\lambda_{0}$. Moreover, under the condition $n \Delta_{n}^{3} \rightarrow 0$ it is asymptotically normal, i.e.

$$
\sqrt{n \Delta_{n}}\left(\lambda_{n}^{*}-\lambda_{0}\right) \stackrel{d}{\rightarrow} N\left(0, \frac{6}{5} \lambda_{0}\right)
$$

Proof. We use the same notation introduced above. By Taylor's formula and the tightness of the sequence $\left\{\check{\lambda}_{n}\right\}$, we have

$$
V_{n}=g_{n}\left(\check{\lambda}_{n}\right)=v^{2}\left(1-\frac{2}{3} \check{\lambda}_{n} \Delta_{n}+O_{p}\left(\Delta_{n}^{2}\right)\right) .
$$

Therefore,

$$
\check{\lambda}_{n}+O_{p}\left(\Delta_{n}\right)=\frac{3}{2 \Delta_{n}}\left(1-\frac{V_{n}}{v^{2}}\right)=\lambda_{n}^{*},
$$

which completes the proof.

\section{An asymptotically efFicient estimator}

In the previous section we have seen that the estimators $\lambda_{n}^{*}$ and $\check{\lambda}_{n}$ are not efficient because their asymptotic variance is $\frac{6}{5} \lambda$ which is strictly greater than $\lambda$. We now present an asymptotically efficient estimator. Consider the following statistic:

$$
\tilde{\lambda}_{n}=\frac{1}{n \Delta_{n}} \sum_{i=1}^{n} \mathbf{1}_{\left\{\left|\eta_{i}\right|<v \Delta_{n}\right\}}=\frac{1}{n \Delta_{n}} \sum_{i=1}^{n} \mathbf{1}_{\left\{N\left(\left[t_{i-1}, t_{i}\right)\right) \geq 1\right\}} .
$$

The statistic $\tilde{\lambda}_{n}$ is not a good estimator of $\lambda$ for fixed $\Delta_{n}$. Indeed,

$$
\mathrm{E}_{0}\left\{\tilde{\lambda}_{n}\right\}=\frac{1}{n \Delta_{n}} \sum_{i=1}^{n} \mathrm{E}_{0}\left\{\mathbf{1}_{\left\{\left|\eta_{i}\right|<v \Delta_{n}\right\}}\right\}=\frac{1-e^{-\lambda_{0} \Delta_{n}}}{\Delta_{n}} .
$$

Instead, we propose the following estimator:

$$
\hat{\lambda}_{n}=-\frac{1}{\Delta_{n}} \log \left(1-\Delta_{n} \tilde{\lambda}_{n}\right),
$$

and Theorem 4.1 proves that it is the efficient estimator in this context.

Theorem 4.1. Let $\Delta_{n} \rightarrow 0, n \Delta_{n} \rightarrow \infty$ as $n \rightarrow \infty$. Then the estimator $\hat{\lambda}_{n}$ in (21) is consistent, asymptotically normal, and attains the minimal variance, i.e. it is asymptotically efficient:

$$
\sqrt{n \Delta_{n}}\left(\hat{\lambda}_{n}-\lambda_{0}\right) \stackrel{d}{\longrightarrow} N\left(0, \lambda_{0}\right) .
$$

Proof. In order to prove consistency and asymptotic normality of $\hat{\lambda}_{n}$ we first prove the same properties for $\tilde{\lambda}_{n}$. Let us consider the following quantity:

$$
\begin{aligned}
U_{n} & =\sqrt{n \Delta_{n}}\left(\tilde{\lambda}_{n}-\mathrm{E}_{0}\left\{\tilde{\lambda}_{n}\right\}\right) \\
& =\frac{1}{\sqrt{n \Delta_{n}}} \sum_{i=1}^{n}\left\{\mathbf{1}_{\left\{\left|\eta_{i}\right|<v \Delta_{n}\right\}}-\mathrm{E}_{0}\left\{\mathbf{1}_{\left\{\left|\eta_{i}\right|<v \Delta_{n}\right\}}\right\}\right\} \\
& =\frac{1}{\sqrt{n \Delta_{n}}} \sum_{i=1}^{n}\left\{\mathbf{1}_{\left\{\left|\eta_{i}\right|<v \Delta_{n}\right\}}-\left(1-e^{-\lambda_{0} \Delta_{n}}\right)\right\}=\sum_{i=1}^{n} \xi_{i}
\end{aligned}
$$

with

$$
\xi_{i}=\frac{1}{\sqrt{n \Delta_{n}}}\left\{\mathbf{1}_{\left\{\left|\eta_{i}\right|<v \Delta_{n}\right\}}-\left(1-e^{-\lambda_{0} \Delta_{n}}\right)\right\}
$$


We have that $\mathrm{E}_{0}\left\{\xi_{i}\right\}=0$; thus $\mathrm{E}_{0}\left\{U_{n}\right\}=0$. Moreover,

$$
\begin{aligned}
n \Delta_{n} \mathbf{V}_{0}\left\{\xi_{i}\right\} & =\mathbf{V}_{0}\left\{\mathbf{1}_{\left\{\left|\eta_{i}\right|<v \Delta_{n}\right\}}\right\} \\
& =\mathrm{E}_{0}\left\{\mathbf{1}_{\left\{\left|\eta_{i}\right|<v \Delta_{n}\right\}}\right\}\left(1-\mathrm{E}_{0}\left\{\mathbf{1}_{\left\{\left|\eta_{i}\right|<v \Delta_{n}\right\}}\right\}\right) \\
& =\left(1-e^{-\lambda_{0} \Delta_{n}}\right) e^{-\lambda_{0} \Delta_{n}} \\
& =\lambda_{0} \Delta_{n}+o\left(\lambda_{0} \Delta_{n}\right)
\end{aligned}
$$

hence

$$
\mathbf{V}_{0}\left\{U_{n}\right\}=\frac{1}{n \Delta_{n}} n\left(\lambda_{0} \Delta_{n}+o\left(\lambda_{0} \Delta_{n}\right)\right)=\lambda_{0}+o(1) .
$$

Finally, the random variables $\xi_{i}$ are independent because they only involve the absolute value of the increments $\eta_{i}$. Since $\left|\xi_{i}\right| \leq 1 / \sqrt{n \Delta_{n}}$, the Lindeberg condition easily follows:

$$
\sum_{i=1}^{n} \mathrm{E}_{0}\left\{\mathbf{1}_{\left\{\left|\xi_{i}\right| \geq \epsilon\right\}} \xi_{i}^{2}\right\} \rightarrow 0
$$

and thus

$$
U_{n} \stackrel{d}{\rightarrow} N\left(0, \lambda_{0}\right)
$$

Now we need to prove the asymptotic normality of $\hat{\lambda}_{n}$ in (21). Let

$$
f_{n}(u)=-\frac{1}{\Delta_{n}} \log \left(1-u \Delta_{n}\right) .
$$

Then

$$
\begin{gathered}
f_{n}^{\prime}(u)=\frac{d}{d u} f_{n}(u)=\frac{1}{1-u \Delta_{n}} \\
\hat{\lambda}_{n}=f_{n}\left(\tilde{\lambda}_{n}\right)
\end{gathered}
$$

and

$$
\lambda_{0}=f_{n}\left(\tilde{\lambda}_{0}\right), \quad \text { where } \tilde{\lambda}_{0}=\frac{1-e^{-\lambda_{0} \Delta_{n}}}{\Delta_{n}} \equiv \mathrm{E}_{0}\left\{\tilde{\lambda}_{n}\right\}
$$

By using the $\delta$-method, we obtain

$$
\begin{aligned}
\sqrt{n \Delta_{n}}\left(\hat{\lambda}_{n}-\lambda_{0}\right) & =\sqrt{n \Delta_{n}}\left(f_{n}\left(\tilde{\lambda}_{n}\right)-f_{n}\left(\tilde{\lambda}_{0}\right)\right) \\
& =\sqrt{n \Delta_{n}}\left(\tilde{\lambda}_{n}-\tilde{\lambda}_{0}\right) f_{n}^{\prime}\left(\tilde{\lambda}_{0}\right)+o_{p}\left(\sqrt{n \Delta_{n}}\left|\tilde{\lambda}_{n}-\tilde{\lambda}_{0}\right|\right) \\
& =U_{n} \frac{1}{1-\lambda_{0} \Delta_{n}}+o_{p}(1)
\end{aligned}
$$

hence

$$
\sqrt{n \Delta_{n}}\left(\hat{\lambda}_{n}-\lambda_{0}\right) \stackrel{d}{\rightarrow} N\left(0, \lambda_{0}\right)
$$

\section{BIBLIOGRAPHY}

1. F. Black and M. S. Scholes, The pricing of options and corporate liabilities, Journal of Political Economy 81 (1973), 637-654.

2. A. De Gregorio and S. M. Iacus, Parametric estimation for the standard and the geometric telegraph process observed at discrete times, Unimi Research Papers, http://services.bepress. com/unimi/statistics/art14 (2006).

3. A. Di Crescenzo and B. Martinucci, On the effect of random alternating perturbations on hazard rates, Scientiae Mathematicae Japonicae 64 (2006), no. 2, 381-394. MR2254153

4. A. Di Crescenz and F. Pellerey, On prices' evolutions based on geometric telegrapher's process, Applied Stochastic Models in Business and Industry 18 (2002), 171-184. MR.1907356 (2003d:60130)

5. G. B. Di Masi, Y. M. Kabanov, and W. J. Runggaldier, Mean-variance hedging of options on stocks with Markov volatilities, Theory of Probability and its Applications 39 (1994), 172-182. MR 1348196 (96h:90020) 
6. D. Florens-Zmirou, Approximate discrete time schemes for statistics of diffusion processes, Statistics 20 (1989), 547-557. MR.1047222 (91e:62215)

7. S. K. Fong and S. Kanno, Properties of the telegrapher's random process with or without a trap, Stochastic Processes and their Applications 53 (1994), 147-173. MR.1290711 (95g:60089)

8. S. Goldstein, On diffusion by discontinuous movements and the telegraph equation, The Quarterly Journal of Mechanics and Applied Mathematics 4 (1951), 129-156. MR0047963 (13:960b)

9. E. E. Holmes, Is diffusion too simple? Comparisons with a telegraph model of dispersal, American Naturalist 142 (1993), 779-796.

10. E. E. Holmes, M. A. Lewis, J. E. Banks, and R. R. Veit, Partial differential equations in ecology: Spatial interactions and population dynamics, Ecology 75 (1994), no. 1, 17-29.

11. S. M. Iacus, Statistical analysis of the inhomogeneous telegrapher's process, Statistics and Probability Letters 55 (2001), no. 1, 83-88. MR1860195 (2002g:60161)

12. M. Kac, A stochastic model related to the telegrapher's equation, Rocky Mountain Journal of Mathematics 4 (1974), 497-509. MR.0510166 (58:23185)

13. Yu. A. Kutoyants, Statistical Inference for Spatial Poisson Processes, Lecture Notes in Statistics, Springer-Verlag, 1998. MR,1644620 (99k:62149)

14. C. Mazza and D. Rullière, A link between wave governed random motions and ruin processes, Insurance: Mathematics and Economics 35 (2004), 205-222. MR2095886 (2005h:60125)

15. R. C. Merton, Theory of rational option pricing, Bell Journal of Economics and Management Science 4 (1973), no. 1, 141-183. MR0496534 (58:15058)

16. E. Orsingher, Hyperbolic equations arising in random models, Stochastic Processes and their Applications 21 (1985), 49-66. MR834990 (87f:35145)

17. E. Orsingher, Probability law, flow function, maximum distribution of wave-governed random motions and their connections with Kirchoff's laws, Stochastic Processes and their Applications 34 (1990), 49-66. MR 1039562 (91g:60086)

18. M. Pinsky, Lectures on Random Evolution, World Scientific, River Edge, New Jersey, 1991. MR.1143780 (93b:60160)

19. N. Ratanov, A jump telegraph model for option pricing, Quantitative Finance 7 (2007), no. 5, 575-583. MR 2358921 (2008m:60167)

20. N. Ratanov, Quantile hedging for telegraph markets and its applications to a pricing of equitylinked life insurance contracts, Borradores de Investigatión, no. 62, apr. 2005, http://www. urosario.edu.co/FASE1/economia/documentos/pdf/bi62.pdf

21. W. Stadje and S. Zacks, Telegraph processes with random velocities, Journal of Applied Probability 41 (2004), 665-678. MR2074815 (2005g:60116)

22. Y. Yao, Estimation of noisy telegraph process: Nonlinear filtering versus nonlinear smoothing, IEEE Transactions on Information Theory 31 (1985), no. 3, 444-446. MR794444 (86f:94007)

23. N. Yoshida, Estimation for diffusion processes from discrete observation, Journal of Multivariate Analysis 41 (1992), no. 2, 220-242. MR.1172898 (93g:62113)

Department of Economics, Business and Statistics, University of Milan, Via ConservatoRio 7, 20122 Milan, Italy

E-mail address: stefano.iacus@unimi.it

Graduate School of Mathematical Sciences, University of Tokyo, 3-8-1 Komaba, MeGUROKU, TOKYO 153-8914, JAPAN

E-mail address: nakahiro@ms.u-tokyo.ac.jp

Received 28/DEC/2006

Originally published in English 